\title{
Verbessern Antioxidanzien den Verlauf?
}

Fragestellung: Aus Zwillingsstudien wissen wir, dass etwa $70 \%$ des Risikos an amyotropher Lateralsklerose (ALS) zu erkranken auf genetischen Faktoren beruhen und etwa $30 \%$ auf Umweltfaktoren zurückzuführen sind.

Hintergrund: In dieser Assoziationsstudie sollte der Zusammenhang zwischen Ernährung und etablierten Funktionsscores für die ALS sowie der respiratorischen Funktion bei Beginn der Erkrankung untersucht werden.

Patienten und Methodik: Über fünf Jahre (2008 bis 2013) wurde in den USA eine Kohortenstudie durchgeführt, die das Ziel hatte, die Rolle von oxidativem Stress bei 302 Patienten, die weniger als 18 Monate an ALS litten, zu definieren. Der Konsum an Nahrungsmitteln wurde mit einem Score (Block Food Frequency Questionnaire, FFQ) gemessen. Die Endpunkte waren die revidierte ALS Functional Rating Scale (ALS-FRS-R) sowie die forcierte Vitalkapazität (FVC).

Nieves JW, Gennings C, FactorLitvak P et al. Association between dietary intake and function in amyotrophic lateral sclerosis. JAMA Neurol 2016; 73 : $1425-32$
Ergebnisse: Es wurde bei 302 Patienten mithilfe einer Regressionsanalyse gezeigt, dass eine höhere Einnahme von Antioxidanzien und Carotenen mit höheren ALSFRS-R-Scores, das heißt einem besseren klinischen

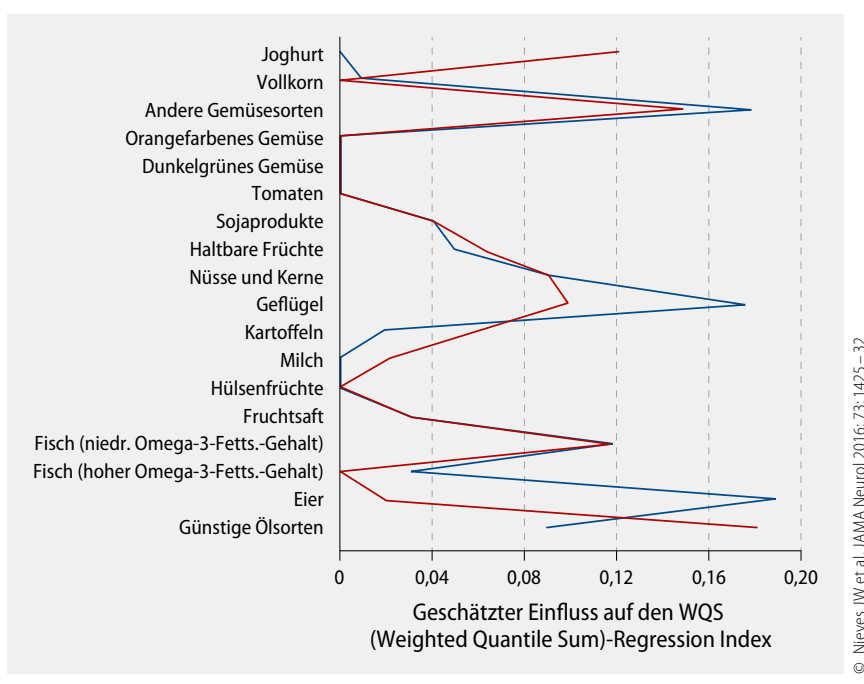

1 Als „gut“ identifizierte Nahrungsmittelgruppen.

Bild, und einer höheren Vitalkapazität zu Beginn der Erkrankung verknüpft waren ( Abb. 1).

Schlussfolgerung: Die Autoren folgern, dass eine erhöhte Aufnahme von Antioxidanzien, Carotenen, Obst und Gemüse zu einem besseren ALS-Verlauf führen könnte.

\section{- Kommentar von Albert C. Ludolph, Ulm}

\section{Die Schlussfolgerung ist gewagt}

Eine interessante Studie, die allerdings zunächst durch einige Möglichkeiten des Bias in ihrer Interpretation getrübt ist. Ein möglicher - nicht untersuchter - Faktor, der die Interpretation des Ergebnisses beeinträchtigen könnte, ist ein besserer sozialer Status, der mit einer besseren Ernährung verknüpft sein sollte, und gleichzeitig zu einer höheren Akzeptanz von symptomatischen therapeutischen Maßnahmen und damit zu einem besseren Verlauf führen sollte. Daher bleibt offen, ob dieses Ergebnis nicht durch Sekundärfaktoren beeinflusst wird. Darüber hinaus haben die Autoren einmalig Funktionsparameter gemessen. Sie weisen zwar darauf hin, dass ausschließlich Longitudinalstudien entscheiden, schließen aber, dass Patienten mit ALS am besten mit Antioxidanzien ernährt werden sollten. Der Hinweis ist sehr richtig, der Schluss ebenso gewagt. Einzig placebokontrollierte, doppelblinde, randomisierte, prospektive Studien können die Frage, ob Antioxidanzien einen Effekt auf den Verlauf der ALS haben, entscheiden.

Am Rande sei erwähnt, dass eine Studie aus den 1990er-Jahren mit dem Antioxidans N-Acetylcystein bei einer kleinen Zahl von Patienten einen vergleichsweise positiven Effekt auf den Verlauf der ALS hatte [1]; aus meiner Sicht sollte diese Studie wiederholt werden (mit einer größeren Zahl von Patienten). Dagegen war die Studie von Ascherio, die 2005 publiziert wurde und einen positiven Effekt von Vitamin E als Prophylaktikum zeigte, nicht reproduzierbar [2]. Auch eine Interventionsstudie mit Vitamin E blieb ohne therapeutischen Effekt.
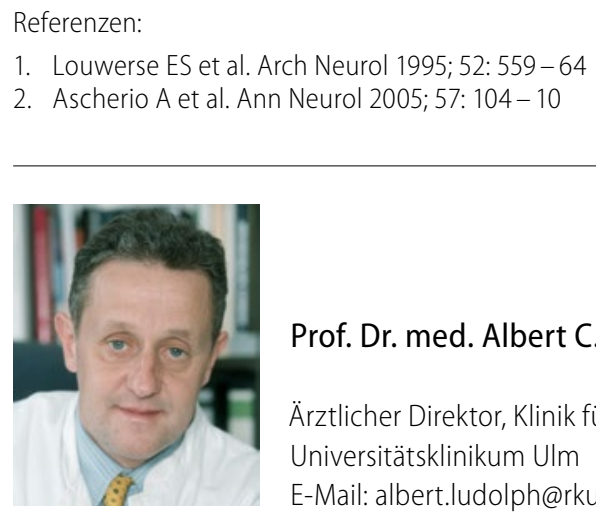

Prof. Dr. med. Albert C. Ludolph, Ulm

Ärztlicher Direktor, Klinik für Neurologie, Universitätsklinikum UIm E-Mail: albert.ludolph@rku.de 